\title{
Activité nitrate réductase in vitro de jeunes plantules de blé (Triticum aestivum $L$ ) cultivées dans les conditions de détermination de la faculté germinative et après amélioration de la nutrition et de l'éclairement
}

\author{
O Bazzigalupi ${ }^{1 *}$, ME Deroche $2^{\star \star *}$, JC Lescure ${ }^{2}$, C Bachelier, S Tardif \\ 1 INRA, domaine de la Minière, station nationale d'essais de semences (SNES), 78280 Guyancourt; \\ 2 INRA, Centre de Versailles, laboratoire du métabolisme et de la nutrition des plantes, 78026 Versailles Cedex, Franice
}

(Reçu le 5 février 1992; accepté le 7 juillet 1992)

\begin{abstract}
Résumé - Quand les plantules de blé croissent dans les conditions de détermination de la faculté germinative édictées par l'ISTA (International Seed Testing Association) : sable humidifié par de l'eau déminéralisée, $8 \mathrm{~h}$ par jour de lumière faible : $55 \mu \mathrm{mol}$ photon. $\mathrm{m}^{-2} . \mathrm{s}^{-1}$, «milieu clos", leur activité nitrate réductase est très faible. En absence de nitrate, il peut s'agir d'une nitrate réductase constitutive, qui varie peu entre 3 et 12 j de croissance. La présence de $\mathrm{KNO}_{3}$ dans le milieu induit l'activité nitrate réductase qui augmente entre 3 et 6 jours, surtout dans les feuilles, puis reste constante et diminue entre 9 et 12 j; dans ce "milieu clos"; l'augmentation de l'intensité lumineuse, à $200 \mu$ mol photon. $\mathrm{m}^{-2} . \mathrm{s}^{-1}$, c'est-à-dire au-dessus du point de compensation en lumière, ne provoque pas l'accroissement de l'activité nitrate réductase que l'on observe si les plantules croissent en "milieu ouvert» : dans ce cas, la transpiration est possible et l'apport de nitrate de potassium ou mieux, de solution nutritive équilibrée, est journalier. Trois jours après le semis, l'activité nitrate réductase dans les racines correspond à plus de la moitié de l'activité totale des plantules; dès le 4 e j, l'activité nitrate réductase dans la partie aérienne dépasse $50 \%$ de l'activité totale; elle atteint $90 \%$ au $6^{e}$ j quel que soit le traitement.
\end{abstract}

activité nitrate réductase in vitro / Triticum aestivum / faculté germinative / intensité lumineuse / nutrition équilibrée / milieu clos / milieu ouvert

\begin{abstract}
Summary - In vitro nitrate reductase activity of wheat (Triticum aestivum $L$ ) seedlings grown under various nutrient and light conditions. When wheat seedlings were grown in conformity with ISTA (the International Seed Testing Association) rules ie on sand, deionized water, in a "closed environment" and at a light intensity of $55 \mu$ mol photon. $m^{-}$ $2 . s^{-1}$, nitrate reductase activity was very low as a constitutive form. Potassium nitrate induced nitrate reductase activity, which increased from the 3rd to the 6th d of growth, mainly in the leaves, then remained constant and decreased from the 9th to the 12th $d$. With a "closed environment", increasing irradiation up to $200 \mu$ mol photon. $m^{-2} . s^{-1}$, ie above the light compensation point, did not induce an increase in enzyme activity. In contrast, when seedlings grew in an "open environment", allowing transpiration and with a daily supply of potassium nitrate or better still a complete nutrient solution, activity was very significant. Three $d$ after sowing, enzyme activity was essentially located in the roots. After $4 d$ of growth, shoot nitrate reductase activity rose to $>50 \%$ and attained $90 \%$ of whole plant activity on the 6 th $d$.
\end{abstract}

in vitro nitrate reductase activity / Triticum aestivum / germinative potential / light intensity / equilibrated nutrition / closed environment / open environment

\footnotetext{
* Adresse actuelle: INTA, Estacion experimental agropecuaria, CC 312700 Pergamino, Argentina

"* Correspondance et tirés à part
} 


\section{INTRODUCTION}

Les agriculteurs souhaitent que chacun des grains mis en terre donne une plante parfaite. Autrefois, la qualité d'un lot de semences se jugeait à son aspect au creux de la main. Aujourd'hui, des techniques permettent d'apprécier la faculté germinative des semences mises sur le marché. Dans le cadre des échanges commerciaux internationaux, I'Association internationale d'essais de semences (ISTA, 1985) a édicté des règles adoptées dans les différents pays. Bien que l'objectif final soit la "détermination de l'aptitude à évoluer en plantes satisfaisantes dans un sol de bonne qualité et dans les conditions favorables d'humidité, de température et de lumière", les règles ISTA préconisent l'emploi de substrat inerte (papier filtre ou sable) arrosé avec de l'eau désionisée ou distillée, donc en l'absence de tout apport minéral; la seule dérogation admise est l'emploi de $\mathrm{KNO}_{3} \quad(0,2 \%)$ quand les semences sont dormantes. Ces expérimentations sont le plus souvent faites à l'obscurité.

Dans le laboratoire SNES (Station nationale d'essais de semences), la faculté germinative est déterminée dans des conditions qui respectent ces règles internationales (sable, éclairement faible de $55 \mu \mathrm{mol}$ photon. $\mathrm{m}^{-2} \cdot \mathrm{s}^{-1}, 8 \mathrm{~h} / \mathrm{j}$ ). Ces règles reposent sur le principe qu'une semence possède en elle-même tout ce qui lui est nécessaire pour germer quand on lui donne de l'eau. On ne connaît pas grand-chose au sujet de l'autonomie des semences vis-à-vis des éléments minéraux. C'est dans ce but que Bazzigalupi (1991) a comparé l'emploi d'eau désionisée à celui d'une solution de $\mathrm{KNO}_{3}(0,2 \%)$. Tout d'abord, l'eau favorise le développement des plantules de blé jusqu'à 3 j, stade où le coléoptile émerge du sable. Ensuite le nitrate de potassium favorise l'utilisation des réserves et le développement de l'appareil photosynthétique. La conclusion de la comparaison entre les effets de $\mathrm{KNO}_{3}$, $\mathrm{KCl}$ et $\mathrm{NaNO}_{3}$ est que le nitrate améliore la mobilisation des réserves davantage que le chlorure, et que le le potassium provoque une meilleure utilisation des substrats (réserves, éléments nutritifs, $\mathrm{CO}_{2}$ ) que le le sodium.

Selon Pinto Contreras et Gaudillère (1987), le passage de l'hétérotrophie à l'autotrophie des jeunes plantules de blé vis-à-vis du carbone se situe entre le $9^{e}$ et le $13^{e} \mathrm{j}$; les réserves du grain servent à la synthèse de squelettes carbonés et les photosynthétats fournissent les substrats pour la production d'énergie. Dans le présent article, les mesures d'échanges gazeux de plantules de blé cultivées avec de l'eau ou du $\mathrm{KNO}_{3}$ dans les conditions SNES permettent de savoir si la photosynthèse est supérieure à la respiration.

De plus, le blé se comporte différemment du soja et du haricot en présence de $\mathrm{KNO}_{3}$ (Bazzigalupi, 1991) : alors que l'accroissement de la teneur en azote total des plantules de haricot et de soja n'est dû qu'à l'augmentation de leur azote nitrique, l'azote réduit des plantules de blé augmente aussi. Cela nous a conduits à examiner le fonctionnement de la nitrate réductase.

Au cours de ces dernières années, la structure et la fonction de la nitrate réductase ont été très étudiées au niveau moléculaire (Caboche et Rouzé, 1990); cette enzyme est inductible par son substrat (Hewitt et Cutting, 1979) bien que I'on connaisse une forme "constitutive" (Andrews et al, 1990); on sait que la lumière contrôle son activité par l'intermédiaire du phytochrome. De plus, les métabolites issus de la photosynthèse sont indispensables au fonctionnement de l'enzyme et utilisés directement ou après transport dans les organes non photosynthétiques (Srivastava, 1980; Lee, 1980; Abrol et al, 1983; Duke et Duke, 1984). La lumière améliore d'abord l'absorption de nitrate chez les jeunes plantules d'orge et d'avoine, puis induit la nitrate réductase (Chen et Ries, 1969); la réduction du nitrate plus rapide à la lumière qu'à l'obscurité est attribuée à la fourniture de produits de la photosynthèse chez l'orge jeune (Aslam et al, 1979). La limitation de la photosynthèse, par privation de $\mathrm{CO}_{2}$, chez des plantules de maïs âgées de $3 \mathrm{j}$, retentit sur l'absorption et la réduction de nitrate (Pace et al, 1990); cette régulation se fait par la fourniture d'énergie et de squelettes carbonés; Deroche (1983) a résumé les relations entre métabolisme carboné et azoté.

Dans la plupart des plantes, l'activité nitrate réductase la plus élevée se trouve dans les feuilles mais elle peut être importante dans les racines après une carence en azote (Talouizte et al, 1984) ou en conditions de nutrition faible en nitrate (Andrews, 1986; Wallace, 1986; Rodgers et Barneix, 1988).

Parmi les études antérieures faites au cours de la germination et des stades jeunes, nous avons retenu celles qui concernent l'activité in vitro chez le blé et quelques graminées. Disa et al $(1982,1985)$ ont trouvé une activité nitrate réductase dans les embryons de blé germant en 
présence de $60 \mathrm{mmol}^{-1} \mathrm{I}^{-1} \mathrm{KNO}_{3}$, après une phase de latence de $20 \mathrm{~h}$ nécessaire à la mise en route des synthèses protéiques; le nitrate s'accumule dans l'embryon mais n'induit par l'enzyme peridant ces 20 premières h (Gupta et al, 1983); chez l'orge, on peut supprimer cette phase de latence par ablation de l'embryon (Gupta et al, 1988).

Le travail présenté ici commence par un rappel des résultats de Bazzigalupi (1991) qui ont conduit à mesurer les échanges gazeux et l'activité nitrate réductase des plantules de blé, de manière à savoir si la photosynthèse et la réduction du nitrate fonctionnent dans ces conditions d'essais de semences. Puis l'activité de la nitrate réductase de jeunes plantules cultivées dans les conditions SNES a été comparée à celle de plantules cultivées dans un environnement plus favorable : éclairement plus élevé, emploi de solution nutritive complète.

\section{MATÉRIEL ET MÉTHODES}

\section{Matériel végétal}

Des grains de blé (Triticum aestivum $L$ ) cv Renan ont germé et donné naissance à des plantules que nous avons étudiées entre 3 et $12 \mathrm{j}$ de croissance, en "milieu clos" ou en "milieu ouvert", sous 2 intensités lumineuses, et avec 3 types d'alimentation minérale.

\section{Conditions de culture}

Le «milieu clos» correspond aux conditions SNES de détermination de la faculté germinative : une boîte en matière plastique $(17,5 \times 12 \times 5,5 \mathrm{~cm})$ contient $\mathrm{du}$ sable fin de Fontainebleau dont l'humidité est de $9 \%$ (90 $\mathrm{ml}$ d'eau désionisée pour $1 \mathrm{~kg}$ de sable sec); 50 grains de blé équidistants sont disposés, à $1 \mathrm{~cm}$ de profondeur, de manière à ce que la radicule se développe vers le bas; la boite est enfermée dans un sac plastique transparent gonflé d'air.

Le "milieu ouvert" correspond à l'emploi de terrines $(44 \times 28,5 \times 6,5 \mathrm{~cm})$ contenant $12 \mathrm{~kg}$ de sable de Biot, dans lesquelles on sème 110 grains de blé que l'on arrose une fois par jour. La capacité de rétention d'eau de ce sable grossier est de $600 \mathrm{ml}$ par terrine.

La nutrition présente 3 variantes : eau, solution de $\mathrm{KNO}_{3}$ à $0,2 \%$ (environ $20 \mathrm{mmol}^{-1}$ ) et une solution nutritive complète (SNC) selon Coïc et Lesaint (1975) contenant en macroéléments : $3,8 \mathrm{mmol.1} \mathrm{I}^{-1} \mathrm{KNO}_{3}, 3$ mmol. $\mathrm{I}^{-1} \mathrm{Ca}\left(\mathrm{NO}_{3}\right)_{2}, 0,1 \mathrm{mmol} . \mathrm{I}^{-1} \mathrm{Mg}\left(\mathrm{NO}_{3}\right)_{2}, 2 \mathrm{mmol}$. $I^{-1} \quad \mathrm{NH}_{4} \mathrm{NO}_{3}, \quad 1$ mmol. $\mathrm{I}^{-1} \quad \mathrm{KH}_{2} \mathrm{PO}_{4}, \quad 0,1 \mathrm{mmol} . \mathrm{I}^{-1}$ $\mathrm{K}_{2} \mathrm{HPO}_{4}, 0,75 \mathrm{mmol}^{-1} \mathrm{MgSO}_{4}, 0,2 \mathrm{mmol}^{-1} \mathrm{I}^{-1} \mathrm{NaCl}$ et en micro-éléments : $24 \mu \mathrm{mol}^{-I^{-1}} \mathrm{H}_{3} \mathrm{BO}_{3}, 10 \mu \mathrm{mol}^{-1} \mathrm{I}^{-1}$ FeEDTA, $10 \mu \mathrm{mol}^{-1} \mathrm{MnSO}_{4}, 3 \mu \mathrm{mol} \mathrm{I}^{-1} \mathrm{ZnSO}_{4}, 1$ $\mu \mathrm{mol} . \mathrm{I}^{-1} \mathrm{CuSO}_{4}, 0,04 \mu \mathrm{mol} . \mathrm{I}^{-1}\left(\mathrm{NH}_{4}\right)_{6} \mathrm{Mo}_{7} \mathrm{O}_{24}$.

Le «milieu clos" implique un seul apport nutritif au début de l'expérience; chaque plantule dispose, pour $12 \mathrm{j}$, de $36 \mu \mathrm{mol}$ de nitrate (solution $20 \mathrm{mmol}^{-1}{ }^{-1}$ $\mathrm{KNO}_{3}$ ) ou $22 \mu \mathrm{mol}$ de nitrates et de $4 \mu \mathrm{mol}$ d'ammonium (solution nutritive complète). En "milieu ouvert", chaque plante dispose, par j, de $108 \mu \mathrm{mol}$ de nitrate (solution $20 \mathrm{mmol}^{-1} \mathrm{I}^{-1} \mathrm{KNO}_{3}$ ) ou $65 \mu \mathrm{mol}$ de nitrate et de $11 \mu$ mol d'ammonium (SNC).

Les conditions climatiques constantes, en chambres de culture, sont la température : $20^{\circ} \mathrm{C}$ et la durée de l'éclairement : $8 \mathrm{~h} / \mathrm{j}$. Les variantes sont les niveaux d'éclairement: mesurés au niveau du sable où germent les semences, ils sont de 55 (conditions SNES) ou $200 \mu \mathrm{mol}$ photon. $\mathrm{m}^{-2} \cdot \mathrm{s}^{-1}$, à l'intérieur du $\mathrm{sac}$, en "milieu clos" et de $200 \mu \mathrm{mol}$ photon. $\mathrm{m}^{-2} . \mathrm{s}^{-1}$ en "milieu ouvert».

Les divers traitements sont résumés dans le tableau I.

\section{Prélèvements}

Les parties aériennes (feuilles + coléoptiles) et les racines ont été prélevées $4 \mathrm{~h}$ après le début de la photopériode, à l'âge de 3, 6, 9 ou 12 j (“milieu clos", $\mathrm{H}_{2} \mathrm{O}, \mathrm{KNO}_{3}$ ou SNC, $55 \mu \mathrm{mol}$ photon. $\mathrm{m}^{-2} \cdot \mathrm{s}^{-1}$ ou

Tableau I. Différentes conditions de culture des plantules de blé. La durée de la photopériode ( $8 \mathrm{~h}$ ) et la température $\left(20^{\circ} \mathrm{C}\right)$ sont les mèmes dans toutes les expériences.

\begin{tabular}{ccccc}
\hline 55 & $\mathrm{SNC}$ & $\mathrm{KNO}_{3}$ & $\mathrm{~S}$ & $\mathrm{SNC}$ \\
\hline 200 & $\mathrm{KNO}_{3}$ & $\mathrm{SNO}$ & "milieu clos" \\
\hline $\mathrm{KNO}_{3}$ & $\mathrm{SNC}$ & "milieu ouvert»
\end{tabular}


SNC, $200 \mu \mathrm{mol}$ photon. $\left.\mathrm{m}^{-2} \cdot \mathrm{s}^{-1}\right)$, tous les jours de 3 à $12 \mathrm{j}$ (200 $\mu$ mol photon. $\mathrm{m}^{-2} . \mathrm{s}^{-1}$, «milieu clos», $\mathrm{KNO}_{3}$, ou "milieu ouvert" $\mathrm{KNO}_{3}$ ou $\mathrm{SNC}$ ). Les racines sont soigneusement débarrassées du sable par rinçage sous un filet d'eau, puis essuyées avec du papier filtre. La matière fraiche est déterminée sur 2 à 10 répétitions selon le stade. Les échantillons sont fixés dans l'azote liquide et conservés à $-80{ }^{\circ} \mathrm{C}$ jusqu'au moment de la détermination de l'activité nitrate réductase et de la teneur en protéines solubles.

\section{Détermination de l'activité nitrate réductase in vitro et dosage des protéines solubles}

L'activité nitrate réductase in vitro est déterminée par la technique de Moureaux et al (1989) après broyage des échantillons dans $N_{2}$ liquide. L'extraction des protéines solubles, dont la nitrate réductase, se fait à $5{ }^{\circ} \mathrm{C}$ dans un tampon 0,05 mol. $^{-1}$ HEPES $\mathrm{pH} 8,2$ contenant $0,1 \mathrm{~mol}^{-1} \mathrm{I}^{-1} \mathrm{NaCl}, 1 \mathrm{mmol}^{-1}$ EDTA, 2 mmol.1-1 2-mercaptoéthanol, $10 \mu$ mol. I $^{-1}$ FAD, $1 \mu \mathrm{mol} . \mathrm{I}^{-1}$ molybdate de sodium et $1 \mu \mathrm{mol} . \mathrm{I}^{-1}$ leupeptine, inhibiteur des protéases des feuilles ou $1 \mu \mathrm{mol} .1^{-1}$ chymostatine, inhibiteur des protéases des racines (Long et Oaks, 1990) et du Polyclar AT. Après $10 \mathrm{mn}$ de centrifugation $(10000 \mathrm{~g})$, les dosages de l'activité nitrate réductase selon Wray et Filner (1970) et des protéines solubles selon Bradford (1976) sont effectués sur le surnageant. Le milieu réactionnel pour la détermination de l'activité enzymatique est du tampon phosphate $0,1 \mathrm{~mol}^{-1}, \mathrm{pH} 7,5$, dans lequel on ajoute du NADH $\left(1,4 \mathrm{mmol}^{-1}{ }^{-1}\right)$ et du $\mathrm{KNO}_{3}\left(0,1 \mathrm{~mol} . \mathrm{I}^{-1}\right)$. La courbe de référence pour les protéines est obtenue avec de la sérumalbumine bovine. Les activités enzymatiques sont exprimées sur la base de la matière fraîche, ou des protéines solubles, ou par plantule. Les résultats présentés ici sont les moyennes de 3-8 déterminations indépendantes (sauf ceux obtenus avec l'eau et $\mathrm{KNO}_{3}$ en "milieu clos" sous lumière faible où il n'y a eu que 2 répétitions). Les écarts types ont été calculés mais on ne les a représentés que sur 1 ou 2 courbes pour ne pas surcharger les figures.

\section{Mesure des échanges gazeux}

La boîte contenant 50 plantules cultivées dans les conditions SNES avec eau ou $\mathrm{KNO}_{3}$ est débarrassée de son enveloppe plastique et placée dans une cellule d'assimilation balayée par un courant d'air comprimé; l'air est humidifié par barbotage dans l'eau, puis homogénéisé dans la cellule par un ventilateur. L'ensemble est thermostaté. À la sortie de la cellule, une partie de l'air est desséchée avant l'entrée dans le tube de l'analyseur par infrarouge. La concentration en $\mathrm{CO}_{2}$ contenu dans cet air est comparée à celle de l'atmosphère de référence prélevée avant le passage dans la cellule d'assimilation et la différence est enregistrée.
Sur la même boite contenant les plantules (de $6 \mathrm{j}$ ou de 9 j), 3 niveaux d'éclairement sont employés successivement : 0 (obscurité pendant la période de nuit); puis, pendant $4 \mathrm{~h}, 55 \mu \mathrm{mol}$ photon. $\mathrm{m}^{-2} \cdot \mathrm{s}^{-1}$ (éclairement de croissance, obtenu par interposition d'un filtre entre la cellule et la rampe de tubes fluorescents); enfin, pendant $4 \mathrm{~h}, 160 \mu \mathrm{mol}$ photon. $\mathrm{m}^{-2} . \mathrm{s}^{-1}$ (éclairement maximum avec ce dispositif).

\section{RÉSULTATS}

\section{Matière sèche, azote nitrique et réduit, et échanges gazeux en "milieu clos", avec de l'eau ou du $\mathrm{KNO}_{3} 20$ mmol.t', sous $55 \mu \mathrm{mol}$ photon. $\mathrm{m}^{-2} . \mathrm{s}^{-1}$}

Des résultats sur les évolutions en masse fraîche et sèche, en azote total et nitrique, en phosphore et en potassium, de plantules se développant dans les conditions utilisées à la Station nationale d'essais de semences (Bazzigalupi, 1991), on peut retenir que, comparé à l'eau, le $\mathrm{KNO}_{3}$ (20 mmol..$^{-1}$ ) ralentit l'imbibition des grains et la croissance des plantules pendant les 3 premiers jours. Après l'émergence des coléoptiles audessus du sable, la solution de $\mathrm{KNO}_{3}$ augmente l'efficacité d'utilisation des réserves (rapport entre les masses sèches des organes produits et utilisés), stimule le développement de l'axe (feuille + racine) de la plantule, modifie l'architecture de la plantule en favorisant le développement de l'appareil photosynthétique.

Les résultats qui ont servi de base aux études décrites dans cet article sont présentés dans le tableau II, notamment les quantités d'azote nitrique et réduit par plantule. Alors que les grains sont réputés sans nitrate, on en mesure une faible quantité, de l'ordre de $0,1 \%$ de la quantité d'azote réduit. Ces quantités restent évidemment faibles en présence d'eau seule. Avec $\mathrm{KNO}_{3}$, les racines et surtout les feuilles s'enrichissent énormément en nitrate. L'action du $\mathrm{KNO}_{3} 20 \mathrm{mmol}^{-1}{ }^{-1}$ aboutit à un accroissement d'azote réduit dans l'ensemble (feuille + racine) de 41 et $124 \mu \mathrm{g}$ par plantule à 6 et 9 j respectivement.

Les mesures d'échanges gazeux (fig 1) ont montré qu'à l'âge de $6 \mathrm{j}$, le point de compensation lumineux, où l'émission de $\mathrm{CO}_{2}$ par la respiration est égale à l'absorption par la photosynthèse, n'est atteint qu'au-dessus de $100 \mu \mathrm{mol}$ photon. $\mathrm{m}^{-2} \cdot \mathrm{s}^{-1}$; c'est à $9 \mathrm{j}$ seulement que l'intensité lumineuse de croissance peut atteindre le point de compensation. On peut en déduire que 
Tableau II. Évolution de la matière sèche (mg.plantule ${ }^{-1}$,), de l'azote nitrique et de l'azote réduit (azote total - azote nitrique) en $\mu$ g.plantule ${ }^{-1}$ dans les différentes parties des plantules de blé développées dans les conditions de détermination de la faculté germinative employées au SNES ("milieu clos", $8 \mathrm{~h}$ de lumière de $55 \mu \mathrm{mol}$ photon.m- $\mathrm{m}^{-2}, \mathrm{~s}^{-1}$, eau déminéralisée) et dans les mêmes conditions avec remplacement de l'eau par une solution $20 \mathrm{mmol}^{-1} \mathrm{KNO}_{3}$. Les matières sèches sont les moyennes obtenues avec 6 boîtes de 50 plantules et, pour l'azote, les moyennes de 3 répétitions; les écarts types sont inférieurs à $10 \%$ de la valeur de la moyenne (Diff = changement dû au $\mathrm{KNO}_{3}$ ) (D'après Bazzigalupi, 1991). La matière sèche a été déterminée après un séjour de $48 \mathrm{~h}$ des échantillons dans une étuve ventilée à $70^{\circ} \mathrm{C}$. L'azote total a été mesuré automatiquement selon la méthode de Berthelot. L'azote nitrique a été dosé après réduction en nitrite sur une colonne cadmium-cuivre. La quantité d'azote réduit a été obtenue par la différence entre azote total et azote nitrique.

\begin{tabular}{|c|c|c|c|c|c|c|c|c|c|}
\hline \multirow{2}{*}{\multicolumn{2}{|c|}{$\begin{array}{c}\hat{A} g e \\
(j)\end{array}$}} & \multicolumn{3}{|c|}{$\begin{array}{c}\text { Matière sèche } \\
\text { (mg.plantule-1) }\end{array}$} & \multicolumn{2}{|c|}{$\begin{array}{l}\text { Azote nitrique } \\
\left(\mu \text { g.plantule }{ }^{-1}\right)\end{array}$} & \multicolumn{3}{|c|}{$\begin{array}{l}\text { Azote réduit } \\
\left(\mu g . \text { plantule }{ }^{-1}\right)\end{array}$} \\
\hline & & $\mathrm{H}_{2} \mathrm{O}$ & $\mathrm{KNO}_{3}$ & Diff & $\mathrm{H}_{2} \mathrm{O}$ & $\mathrm{KNO}_{3}$ & $\mathrm{H}_{2} \mathrm{O}$ & $\mathrm{KNO}_{3}$ & Diff \\
\hline \multicolumn{10}{|l|}{ Grain } \\
\hline & 0 & 42,9 & 42,9 & 0 & 0,7 & 0,7 & 736,6 & 736,6 & 0 \\
\hline & 3 & 35,9 & 36,6 & $+0,7$ & 1,1 & 1,8 & 528,8 & 573,1 & $+44,3$ \\
\hline & 6 & 21,4 & 21,2 & $-0,2$ & 0,6 & 3,7 & 263,5 & 266,7 & $+3,2$ \\
\hline & 9 & 11,3 & 9,5 & $-1,8$ & 0,3 & 1,7 & 149,7 & 119,2 & $-30,5$ \\
\hline \multicolumn{10}{|l|}{ Racine } \\
\hline & 3 & 2,5 & 2,3 & $-0,2$ & 0,3 & 17,6 & 89,6 & 76,7 & $-12,9$ \\
\hline & 6 & 7,3 & 6,5 & $-0,8$ & 0,6 & 59,4 & 175,7 & 123,3 & $-52,4$ \\
\hline & 9 & 8,7 & 8,6 & $-0,1$ & 0,4 & 69,2 & 176,4 & 149,1 & $-30,3$ \\
\hline \multicolumn{10}{|l|}{ Feuille } \\
\hline & 3 & 1,5 & 1,5 & 0 & 0,1 & 0,7 & 90,1 & 93,3 & $+\quad 3,2$ \\
\hline & 6 & 5,8 & 7,5 & $+1,7$ & 0,3 & 66,7 & 264,5 & 358,3 & $+93,8$ \\
\hline & 9 & 8,9 & 13,5 & $+4,6$ & 0,3 & 162,4 & 391,0 & 542,2 & $+151,2$ \\
\hline \multicolumn{10}{|c|}{ Feuille + racine } \\
\hline & 3 & 4,0 & 3,8 & $-0,2$ & 0,4 & 18,3 & 179,7 & 170,0 & $-9,7$ \\
\hline & 6 & 131,1 & 14,0 & $+0,9$ & 0,9 & 126,1 & 440,2 & 481,6 & $+41,4$ \\
\hline & 9 & 17,6 & 22,1 & $+4,5$ & 0,7 & 231,6 & 567,4 & 691,3 & $+123,9$ \\
\hline \multicolumn{10}{|l|}{ Total } \\
\hline & 0 & 42,9 & 42,9 & 0 & 0,7 & 0,7 & 736,6 & 736,6 & 0 \\
\hline & 3 & 39,9 & 40,4 & $+0,5$ & 1,5 & 20,1 & 708,5 & 743,1 & $+34,6$ \\
\hline & 6 & 34,5 & 35,2 & $+0,7$ & 1,5 & 129,8 & 703,7 & 748,3 & $+44,6$ \\
\hline & 9 & 28,9 & 31,6 & $+2,7$ & 1,0 & 233,3 & 717,1 & 810,5 & $+93,4$ \\
\hline
\end{tabular}

l'activité photosynthétique des plantules de blé cultivées dans les conditions SNES de détermination de leur faculté germinative est faible. L'activité respiratoire des plantules nourries par du $\mathrm{KNO}_{3} 20 \mathrm{mmol}^{-1} \mathrm{I}^{-1}$ est plus grande que celle des plantules alimentées par l'eau seule ( $x 1,7$ à $6 \mathrm{j}, \mathrm{x}$ 1,4 à $9 \mathrm{j}$ ).

On peut calculer le bilan en $\mathrm{CO}_{2}$ de ces plantules poussant avec $8 \mathrm{~h}$ de lumière et $16 \mathrm{~h}$ d'obscurité : on obtient, en mg.plantule $\mathrm{e}^{-1} . \mathrm{j}^{-1}$, des valeurs toujours négatives : $-1,41\left(\mathrm{H}_{2} \mathrm{O}\right)$ et $-1,70$ $\left(\mathrm{KNO}_{3}\right)$ à $6 \mathrm{j} ;-1,25\left(\mathrm{H}_{2} \mathrm{O}\right)$ et $-1,88\left(\mathrm{KNO}_{3}\right)$ à $9 \mathrm{j}$.
Ces valeurs sont proches de celles que l'on peut déduire du tableau II : les pertes de matière sèche entre 3 et $6 j$, et entre 6 et $9 j$ sont comprises entre 1,2 et 1,9 mg.plantule ${ }^{-1} \cdot \mathrm{j}^{-1}$. La quantité de $\mathrm{CO}_{2}$ n'est donc pas le facteur limitant de la photosynthèse dans ce "milieu clos".

Avec cette lumière faible, en présence de $\mathrm{KNO}_{3}$, les feuilles et les racines accumulent beaucoup de nitrate et ont aussi une plus grande quantité d'azote réduit (tableau II), ce qui laisse supposer que la nitrate réductase fonctionne. 


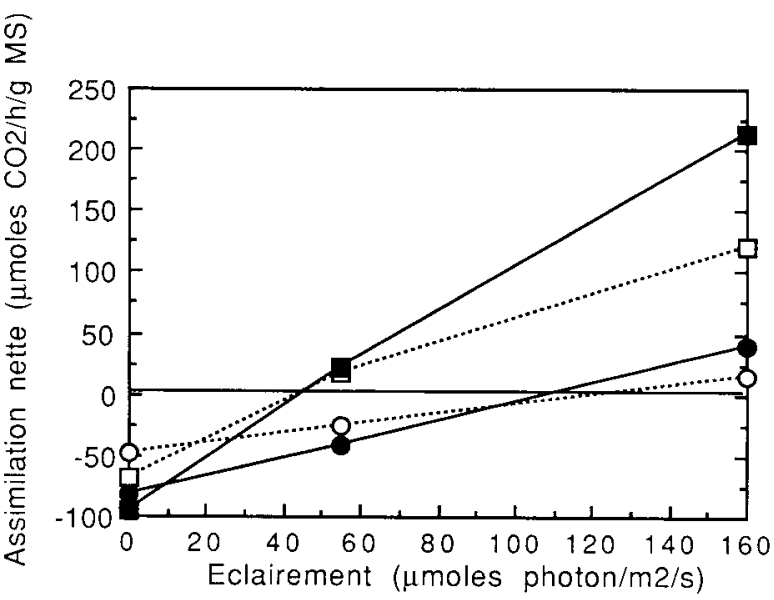

Fig 1. Assimilation nette de $\mathrm{CO}_{2}\left(\mu \mathrm{mol} \cdot \mathrm{h}^{-1} \cdot \mathrm{g}^{-1} \mathrm{MS}\right)$ de plantules de blé, âgées de $6 \mathrm{j}$ (symboles ronds) et de $9 \mathrm{j}$ (symboles carrés), cultivees dans les conditions de détermination de la faculté germinative (milieu clos, $55 \mu$ mol.photon. $\mathrm{m}^{-2} \cdot \mathrm{s}^{-1}$ ), alimentées par l'eau déminéralisée (traits pointillés) ou 20 mmol. I $^{-1} \mathrm{KNO}_{3}$ (traits continus). Les mesures d'échanges gazeux sont faites pendant $4 \mathrm{~h}$ avant le début de la photopériode (éclairement 0 ), puis pendant $4 \mathrm{~h}$ sous l'éclairement de croissance $\left(55 \mu \mathrm{mol}\right.$. photon. $\left.\mathrm{m}^{-2} \cdot \mathrm{s}^{-1}\right)$, puis pendant $4 \mathrm{~h}$ sous un éclairement plus élevé $\left(160 \mu \mathrm{mol}\right.$ photon. $\left.\mathrm{m}^{-2} \cdot \mathrm{s}^{-1}\right)$.

\section{Matière fraîche, protéines solubles et activité nitrate réductase}

\section{"Milieu clos»}

Intensité lumineuse de $55 \mu \mathrm{mol}$ photon. $\mathrm{m}^{-2} \cdot \mathrm{s}^{-1}$, eau, $\mathrm{KNO}_{3}$ ou solution nutritive complète

La matière fraîche des plantules (feuilles et racines) de $3 \mathrm{j}$ est identique quand la lumière est faible quelle que soit l'alimentation. L'apport d'éléments minéraux n'augmente la masse fraîche des racines qu'à $12 \mathrm{j}$ (SNC, $\times 1,2, \mathrm{KNO}_{3}$, $\times 1,4)$. Les parties aériennes réagissent à la présence de $\mathrm{KNO}_{3}$ ou d'autres sels dans la solution nutritive, dès $9 \mathrm{j}(\times 1,75)$, pour atteindre un doublement à $12 \mathrm{j}$ (fig 2 ).

Les teneurs en protéines solubles sont environ 5 fois plus grandes dans les feuilles que dans les racines (fig 2). C'est au stade de 3 jours que I'on a mesuré les valeurs les plus élevées; à ce stade, Bazzigalupi (1991) a montré que la mobilisation des réserves des grains de blé est la même en présence d'eau seule ou d'une solution $20 \mathrm{mmol}^{-1} \mathrm{KNO}_{3}$. Les quantités de protéines solubles par plantule (feuille + racine) mesurées ici sont de $0,2 \mathrm{mg}$ avec l'eau et le $\mathrm{KNO}_{3}^{-}$ et de $0,3 \mathrm{mg}$ si la solution nutritive est complète. Aux stades de 6,9 et $12 \mathrm{j}$ les teneurs en pro- téines solubles diminuent; mais, comme conséquence d'une meilleure croissance, la quantité de protéines solubles par plantule est plus grande quand elles sont alimentées par $\mathrm{KNO}_{3}$ ou la solution nutritive complète ( $x 1,5$ ou $\times 2$, respectivement).

Dans les plantules ne recevant que de l'eau, on a mesuré une activité nitrate réductase in vitro faible, à la limite de la sensibilité de la technique (fig 3); il s'agit peut-être de la nitrate réductase "constitutive". II n'est pas sûr que la très faible quantité de nitrate décelée dans les grains (tableau II) soit analytiquement significative et suffisante pour induire une faible activité enzymatique.

Quand il y a du nitrate dans le milieu nutritif, l'activité nitrate réductase double (parties aériennes) ou triple (racines) dès $3 \mathrm{j}$; à ce stade, tout en restant faible, l'activité dans les racines est égale ou supérieure à celle des feuilles et coléoptiles, notamment si on l'exprime sur la base des protéines solubles. Les activités dans les parties aériennes augmentent rapidement $(x 4)$ entre 3 et $6 \mathrm{j}\left(\mathrm{KNO}_{3}, \mathrm{SNC}\right)$ puis se stabilisent à $9 \mathrm{j}$ et diminuent à $12 \mathrm{j}$, que la base de référence soit la matière fraîche, les protéines solubles ou la plantule entière (fig 3 ). Les activités nitrate réductase des racines ne varient pas de manière significative.

Les activités nitrate réductase sont de 16, 213 et 287 nanomol $\mathrm{NO}_{2}^{-} \cdot \mathrm{h}^{-1}$. [plantule (feuille $+\mathrm{ra}$ cine)] $]^{-1}$ aux stades 3,6 et 9 jours, en présence de $\mathrm{KNO}_{3}$. On peut remarquer que les accroissements en azote réduit (tableau II), sont, de la même manière, plus importants entre 3 et $6 j$ qu'entre 6 et $9 \mathrm{j}$.

Le «milieu clos" et la faible lumière permettent donc à l'activité nitrate réductase de s'exprimer pendant la première partie de la croissance étudiée.

Intensité lumineuse de $200 \mu \mathrm{mol}$

photon. $\mathrm{m}^{-2} \cdot \mathrm{s}^{-1}, \mathrm{KNO}_{3}$

ou solution nutritive complète

Une expérience préliminaire a été faite pendant les mesures d'échanges gazeux. On a constaté que les $8 \mathrm{~h}$ de ventilation (retrait de l'enveloppe plastique) et $4 \mathrm{~h}$ d'éclairement à $160 \mu \mathrm{mol}$ photon. $\mathrm{m}^{-2} \cdot \mathrm{s}^{-1}$ appliquées aux plantules cultivées préalablement sous $55 \mu$ moles photon. $\mathrm{m}^{-2} . \mathrm{s}^{-1}$ n'avaient pas accru leur activité nitrate réductase : pour les feuilles de plantules de $6 \mathrm{j}$, après $4 \mathrm{~h}$ sous $55 \mu \mathrm{mol}$ photon. $\mathrm{m}^{-2}$. $\mathrm{s}^{-1}$, elle est de 2,9 

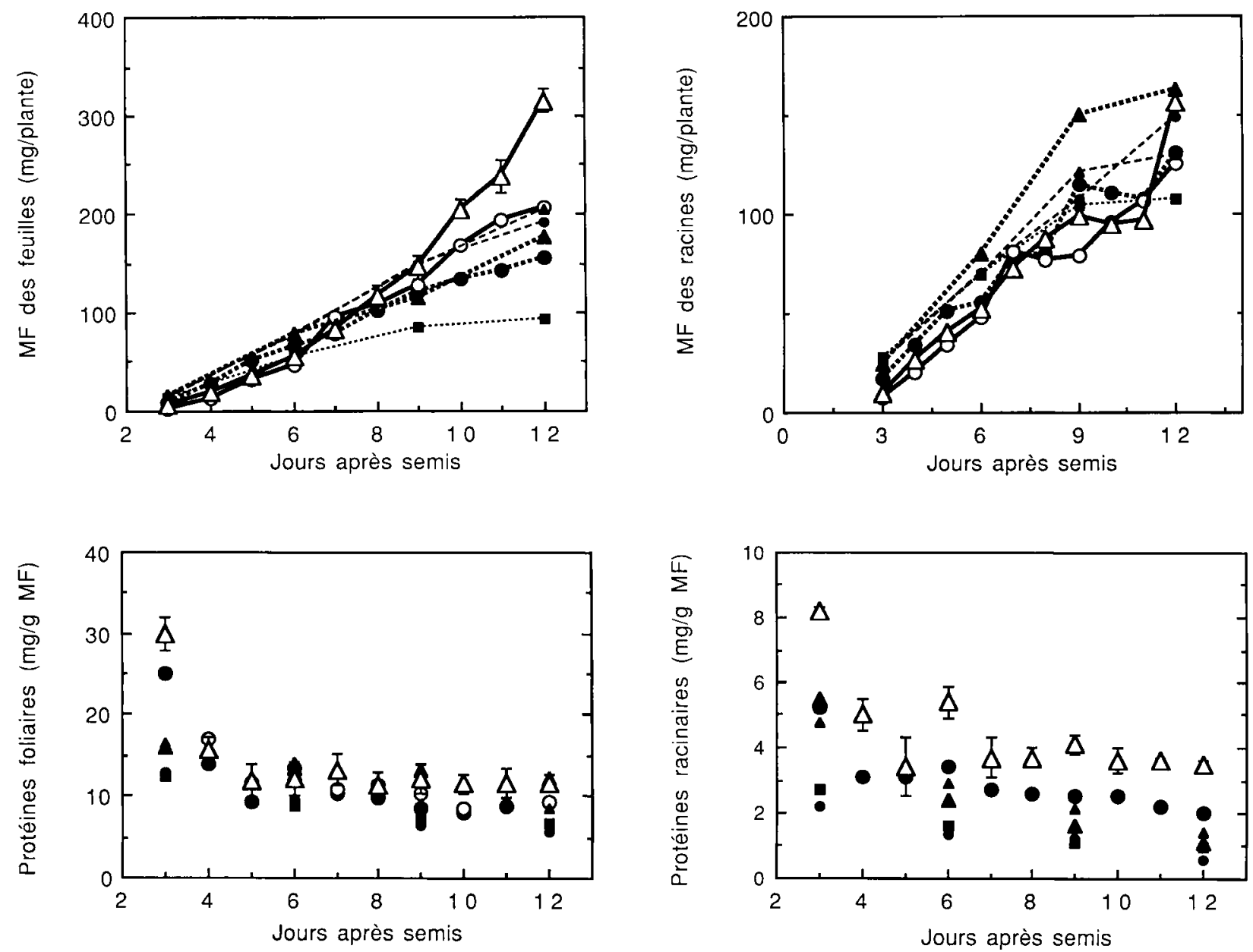

Fig 2. Croissance en matière fraîche (mg.plantule ${ }^{-1}$ ) et teneur en protéines solubles $\left(\mathrm{mg} \cdot \mathrm{g}^{-1} \mathrm{MF}\right.$ ) des parties aériennes et des racines de plantules de blé, sous 2 intensités lumineuses, en "milieu clos" (un seul apport nutritif au semis) et en "milieu ouvert" (apport journalier) et différentes alimentations. Les écarts types ne sont représentés que sur 2 courbes pour ne pas trop surcharger la figure. "Milieu clos", $55 \mu \mathrm{mol}$ photon. $\mathrm{m}^{-2} \cdot \mathrm{s}^{-1}$ (traits discontinus fins), eau déminéralisée (symboles carrés), $20 \mathrm{mmol}^{-1} \mathrm{KNO}_{3}$ (symboles ronds), solution nutritive complète (symboles triangulaires); "milieu clos", $200 \mu$ mol photon. $\mathrm{m}^{-2} \cdot \mathrm{s}^{-1}$ (traits discontinus épais), $20 \mathrm{mmol} . \mathrm{I}^{-1} \mathrm{KNO}_{3}$ (symboles ronds), solution nutritive complète (symboles triangulaires); "milieu ouvert", $200 \mu$ mol photon. $\mathrm{m}^{-2} . \mathrm{s}^{-1}$ (traits continus), $20 \mathrm{mmol}^{-1} \mathrm{KNO}_{3}$ (symboles ronds ouverts), solution nutritive complète (symboles triangulaires ouverts).

$\pm 0,5 \mu$ moles $\mathrm{NO}_{2}^{-} \cdot \mathrm{h}^{-1} \cdot \mathrm{g}^{-1} \mathrm{MF}$; après $4 \mathrm{~h}$ sous $160 \mu \mathrm{mol}$ photon. $\mathrm{m}^{-2}$. $\mathrm{s}^{-1}$ dans la cellule de mesure des échanges gazeux, elle est de $2,9 \pm 0,8$. À l'âge de $9 \mathrm{j}$ et dans les mêmes conditions, les valeurs mesurées sont identiques $(1,5 \pm 0,2 \mu \mathrm{mol}$ $\left.\mathrm{NO}_{2}^{-} \cdot \mathrm{h}^{-1} \cdot \mathrm{g}^{-1} \mathrm{MF}\right)$. Cette remarque est confirmée quelle que soit la base de référence $(6 j: 312 \pm$ 81 et $300 \pm 81 \mu \mathrm{mol} \mathrm{NO}-\mathrm{NO}_{2}^{-1} \cdot \mathrm{g}^{-1}$ protéines solubles, $9 \mathrm{j}: 182 \pm 45$ et $170 \pm 29 \mu \mathrm{mol} \mathrm{NO} \mathrm{N}_{2}^{-} \cdot \mathrm{h}^{-1}$. $\mathrm{g}^{-1}$ protéines solubles). Quelques $\mathrm{h}$ d'intensité lumineuse supérieure au point de compensation lumineux (fig 1) ne modifient pas l'activité nitrate réductase des jeunes plantules de blé cultivées sous $55 \mu \mathrm{mol}$ photon. $\mathrm{m}^{-2} . \mathrm{s}^{-1}$.

On a donc employé une intensité lumineuse de $200 \mu \mathrm{mol}$ photon. $\mathrm{m}^{-2} \cdot \mathrm{s}^{-1}$ pendant toute la croissance.
L'augmentation d'intensité lumineuse ralentit la croissance des plantules alimentées par $\mathrm{KNO}_{3}(\times 0,6$ à $3 \mathrm{j} ; \times 0,8$ à 6,9 et $12 \mathrm{j}$ ) alors que la matière fraîche des plantules (fig 2) ayant reçu de la solution nutritive complète pendant 3 ou $6 \mathrm{j}$ est identique sous 55 et $200 \mu \mathrm{mol}$ photon. $\mathrm{m}^{-2} \cdot \mathrm{s}^{-1}$. Après 9 et $12 \mathrm{j}$ sous $200 \mu \mathrm{mol}$ photon. $\mathrm{m}^{-2} \cdot \mathrm{s}^{-1}$, la matière fraîche des feuilles est moins élevée $(x 0,8)$ et celle des racines, plus grande $(x, 2)$ que sous $55 \mu \mathrm{mol}$ photon. $\mathrm{m}^{-2} \cdot \mathrm{s}^{-1}$.

On observe des teneurs en protéines solubles plus élevées (fig 2) dans les plantules alimentées par $\mathrm{KNO}_{3}$ sous forte lumière $(x 2$ dans les racines des 4 stades étudiés et dans les parties aériennes de $3 \mathrm{j}$ ) quand on les compare aux plantules sous faible intensité lumineuse; la so- 
lution nutritive complète ne provoque pas de changement dans les concentrations en protéines solubles, par rapport au $\mathrm{KNO}_{3}$, mais une augmentation de la quantité de protéines solubles par plantule.

L'augmentation de l'éclairement de 55 à 200 $\mu \mathrm{mol}$ photon. $\mathrm{m}^{-2} . \mathrm{s}^{-1}$ pendant la croissance en "milieu clos" n'a pas d'effet significatif sur l'activité nitrate réductase des plantules de blé
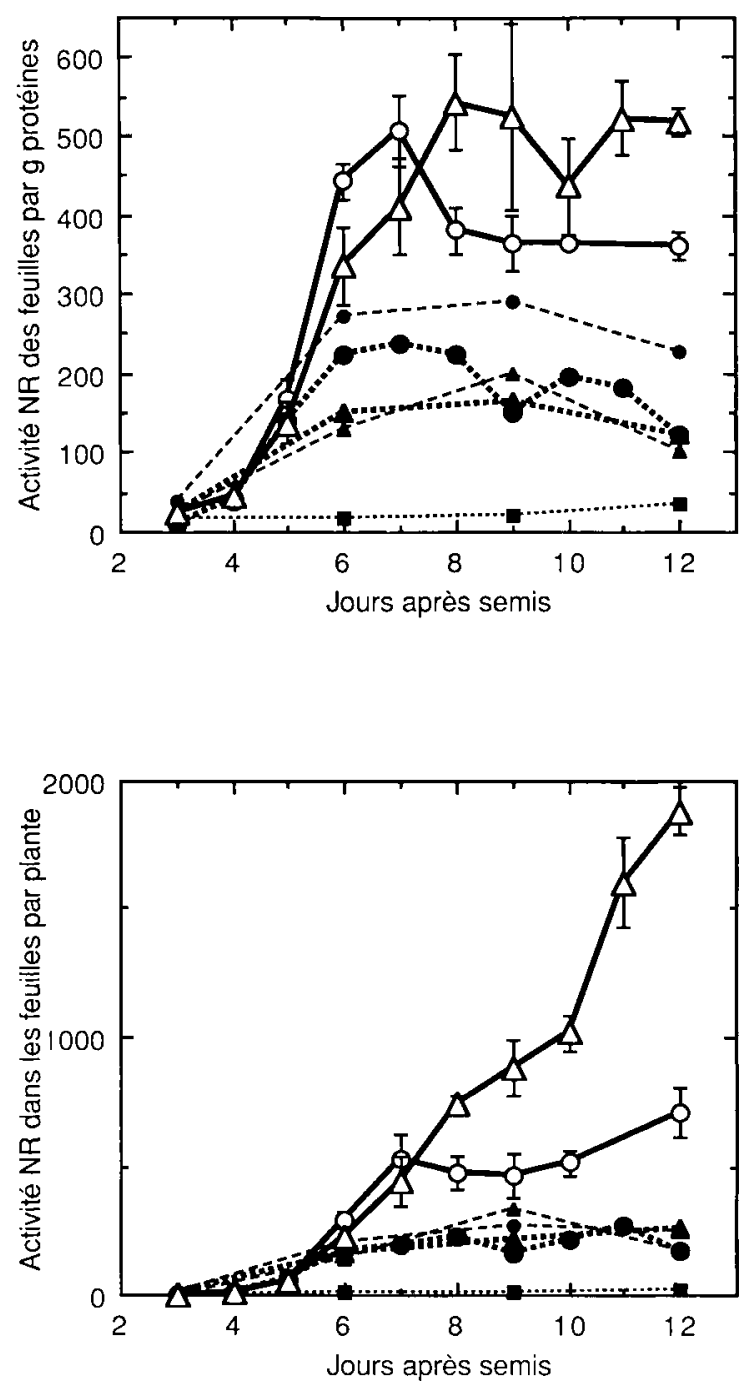

Fig 3. Activité nitrate réductase in vitro ( $\mu$ moles $\mathrm{NO}_{2}^{-} \cdot \mathrm{h}^{-1} \cdot \mathrm{g}^{-1}$ protéines solubles, $\mu$ moles $\mathrm{NO}_{2}^{-} \cdot \mathrm{h}^{-1}$. plantule-1) dans les parties aériennes de plantules de blé croissant sous 2 intensités lumineuses, en "milieu clos" (un seul apport au semis) ou en "milieu ouvert" (apport journalier) et avec différentes alimentations. Pour ne pas surcharger cette figure, nous ne présentons pas les courbes illustrant l'activité sur la base de la matière fraiche car elles sont assez semblables à celles qui la représentent sur la base des proténes solubles : les valeurs de l'axe des ordonnées sont alors comprises entre 0 et 8 $\mu \mathrm{mol} \mathrm{NO} \cdot \mathrm{h}^{-1} \cdot \mathrm{g}^{-1} \mathrm{MF}$, et la courbe correspondant aux plantules en "milieu clos" avec $20 \mathrm{mmol}^{-1} \mathrm{KNO}_{3}$ sous éclairement faible est indiscernable des autres courbes en «milieu clos". Les traits et les symboles sont les mêmes que ceux de la figure 2. qu'elles soient en présence de $20 \mathrm{mmol} . \mathrm{I}^{-1}$ $\mathrm{KNO}_{3}$ ou de solution nutritive complète (fig 3). Cette stagnation de l'activité nitrate réductase des feuilles de plantules cultivées en «milieu clos» est particulièrement nette quand on l'exprime par plantule (fig 3) ou par g de matière fraîche (résultats non montrés). En «milieu clos", l'activité de la nitrate réductase des jeunes plantules de blé n'augmente que de 3 à 6-7 j.

Le facteur limitant ici l'expression de l'activité nitrate réductase ne semble pas être l'intensité lumineuse.

\section{"Milieu ouvert» et intensité lumineuse de $200 \mu$ mol photon. $\mathrm{m}^{-2} . \mathrm{s}^{-1}, \mathrm{KNO}_{3}$ ou solution nutritive complète}

Dans ces conditions, l'apport nutritif est journalier; chaque plantule dispose de 35 fois plus de nitrate au cours des $12 \mathrm{j}$ de croissance qu'en "milieu clos". De plus, la transpiration existe et peut favoriser les mouvements d'eau et de nutriments. Comme précédemment, l'intensité lumineuse permet une assimilation nette positive de $\mathrm{CO}_{2}$.

Cependant, de 3 j à 6 j, la croissance en matière fraîche est plus faible en «milieu ouvert" qu'en «milieu clos» (fig 2).

C'est avec l'arrosage quotidien par la solution nutritive complète (fig 2) que l'on a mesuré les plus grandes teneurs en protéines solubles dans les parties aériennes et les racines au stade de 3 jours. Cependant à cause de la différence de croissance en masse, les quantités de protéines solubles par plantule, aux stades jeunes, sont inférieures en "milieu ouvert» comparé au "milieu clos"; c'est seulement avec la solution nutritive complète apportée chaque jour et au-delà de 10 j que la quantité par plantule se distingue nettement des autres traitements.

Quand les plantules émergent seulement de la surface du sable, l'activité nitrate réductase des racines par $\mathrm{g}$ de matière fraîche ou de protéines solubles est plus faible dans le mode de culture "ouvert" que dans le mode "clos" (avant $6 \mathrm{j}$ pour les feuilles et les racines). Ensuite, au stade $6 \mathrm{j}$, c'est le $\mathrm{KNO}_{3} 20 \mathrm{mmol} . \mathrm{I}^{-1}$ qui provoque la plus grande activité sur la base de la matière fraîche (résultats non montrés), des protéines solubles ou de la plantule entière (fig 3). Quand les plantules croissent de 8 à 12 j avec $\mathrm{KNO}_{3}$ seul, les performances obtenues pour l'activité nitrate 
réductase diminuent par $g$ de matière fraîche, restent stationnaires sur la base des protéines solubles ou augmentent très peu quand elles sont exprimées par plantule. C'est avec la solution nutritive complète apportée quotidiennement, que les potentialités de l'enzyme s'expriment le mieux.

\section{Répartition de la masse fraîche et de l'activité nitrate réductase entre racines et parties aériennes}

Au stade de $3 \mathrm{j}$, la masse de matière fraîche des racines est environ 2 fois plus grande que celle des parties aériennes : la matière fraîche des parties aériennes représente moins de $30 \%$ du total feuilles + racines (arrosage journalier par $20 \mathrm{mmol}^{-1} \mathrm{l}^{-1} \mathrm{KNO}_{3}$ ) alors qu'elle est de $33 \%$ avec l'eau en "milieu clos" et de $36-38 \%$ dans tous les autres cas (fig 4). Ensuite, la partie aérienne devient prédominante surtout en "milieu ouvert». En alimentation hydrique stricte, la proportion de racines est très importante (carence minérale).

La participation des racines à l'activité nitrate réductase totale (feuilles + racines) est très importante à ce stade de 3 j (fig 4 ) où elle se situe entre 50 et $75 \%$ de l'activité totale. Dès $4 j$, l'activité des parties aériennes correspond à la moitié de l'activité totale; à $6 \mathrm{j}$, elle dépasse $80 \%$.

\section{DISCUSSION ET CONCLUSION}

Les règles ISTA qui préconisent l'emploi de l'eau pour la détermination de la faculté germinative des semences semblent bonnes pour des durées très courtes de croissance, n'excédant pas $3 \mathrm{j}$; à ce stade, la racine est plus grande que la feuille encore contenue dans le coléoptile (fig 4); la lumière n'est peut-être pas nécessaire comme l'ont montré des traitements à l'obscurité (Bazzigalupi, 1991). Le confinement empêchant la transpiration conduit à des plantules dont la matière fraîche est plus grande jusqu'à $6 \mathrm{j}$. La croissance en matière fraîche est à peu près la même de 6 à $9 j$ dans les divers traitements. Même en «milieu ouvert", le $\mathrm{KNO}_{3}$ ne provoque pas d'augmentation de la croissance en matière fraîche. Après le $10^{\mathrm{e}} \mathrm{j}$, l'alimentation par la solution nutritive complète améliore la croissance.

Les teneurs en protéines solubles des parties aériennes des plantules de 3 jours, en "milieu clos" sous $55 \mu \mathrm{mol}$ photon. $\mathrm{m}^{-2} . \mathrm{s}^{-1}$, sont semblables avec l'eau ou $\mathrm{KNO}_{3}$. À ce stade, Bazzigalupi (1991) a noté une même mobilisation des réserves estimée par le rapport de la matière sèche produite à la matière sèche consommée. Si on fait l'hypothèse que la teneur en protéines solubles est une image de la mobilisation des réserves des semences, il faut noter, à $3 \mathrm{j}$, une nette augmentation dans les conditions "milieu clos" entre 55 et $200 \mu \mathrm{mol}$ photon. $\mathrm{m}^{-2} \cdot \mathrm{s}^{-1}$ avec
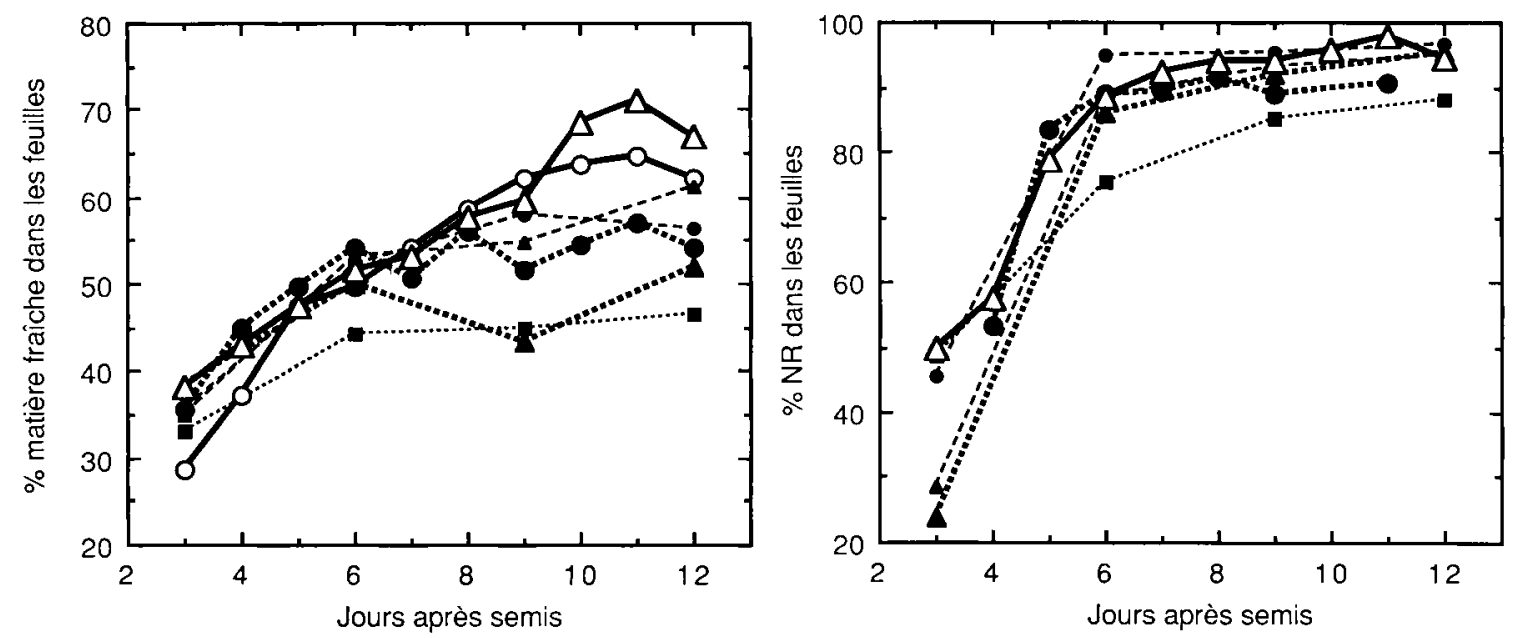

Fig 4. Pourcentages de la matière fraîche et de l'activité nitrate réductase dans les parties aériennes de plantules (feuilles + racines) de blé croissant sous 2 intensités lumineuses, en "milieu clos " (un seul apport au semis) ou en "milieu ouvert" (apport journalier) et avec différentes alimentations. Le pourcentage dû aux racines est le complément à 100 pour chacune des valeurs indiquées dans cette figure. Les traits et les symboles sont les mêmes que ceux de la figure 2. 
$\mathrm{KNO}_{3}$; cet accroissement devient très important à ce stade quand on passe en "milieu ouvert" avec la solution nutritive complète alors qu'on n'en observe pas en «milieu clos». On peut supposer que la meilleure utilisation des réserves due à $\mathrm{KNO}_{3}$ observée par Bazzigalupi (1991) dans les conditions SNES aux stades 6 et 9 j doit commencer beaucoup plus tôt ( $3 \mathrm{j}$ ) avec une intensité lumineuse plus élevée, un "milieu ouvert" et une alimentation équilibrée.

Disa et al (1982, 1985), Gupta et al (1983) ont étudié la nitrate réductase dans les embryons de blé entre 12 et $80 \mathrm{~h}$ de germination; on peut donc comparer leurs observations avec celles présentées ici pour le stade $3 \mathrm{j}$. L'activité nitrate réductase très faible, de l'ordre de $20 \mu \mathrm{mol}$ $\mathrm{NO}_{2}^{-} \cdot \mathrm{h}^{-1} \cdot \mathrm{g}^{-1}$ protéines, que nous avons mesurée dans les plantules alimentées par l'eau (fig 3) n'a pas été détectée par ces auteurs; cette activité est peut-être "constitutive" (Andrews et al, 1990) ou due à la présence d'une très faible quantité de nitrate présent dans les semences (tableau II). Comme Disa et al (1982, 1985), nous avons observé une augmentation importante de l'activité nitrate réductase avec le nitrate et, de plus, la localisation de l'activité nitrate réductase d'abord dans les racines ( $3 \mathrm{j}$ ) puis dans les feuilles; au $4^{\mathrm{e}} \mathrm{j}$, la participation des parties aériennes dépasse $50 \%$ pour atteindre $90 \%$ au $6^{\mathrm{e}}$ j quel que soit le traitement (fig 4). Ce rôle prépondérant des feuilles dans la réduction des nitrates a été souligné chez de nombreuses plantes herbacées (Minotti et Jackson, 1970; Andrews, 1986; Wallace, 1986).

Le «milieu clos» n'empêche pas l'induction de la nitrate réductase par le nitrate. L'activité augmente de 3 à $6 \mathrm{j}$, mais elle ne dépasse jamais celle observée à $6 \mathrm{j}$ quel que soit le niveau de l'éclairement de croissance des jeunes plantules de blé (fig 3).

Le "milieu clos" avec une alimentation uniquement hydrique limite la croissance des plantules; l'apport de nitrate de potassium $20 \mathrm{mmol}^{.1} \mathrm{I}^{-1}$ en «milieu clos» améliore la croissance. On a calculé que chaque plantule dispose d'environ $500 \mu \mathrm{g}$ d'azote; or, en $9 \mathrm{j}$, l'accroissement en azote total (nitrique + réduit) est de l'ordre de $300 \mu \mathrm{g}$ par plantule (tableau II). La première limitation du "milieu clos" est l'alimentation nitrique.

On a indiqué qu'en "milieu ouvert", chaque plantule dispose par jour de plus d'azote qu'en "milieu clos" pour $12 \mathrm{j}$. De plus, l'apport journalier de nitrate en «milieu ouvert" (transpiration, mouvement d'eau et de métabolites vers les feuilles possibles) provoque d'abord à $6 \mathrm{j}$ une nette augmentation de l'activité nitrate réductase des feuilles (fig 3 ); ensuite à $8 j$ et au-delà, les éléments autres que le nitrate de la solution nutritive complète conduisent à de meilleures performances de cette activité enzymatique. Ainsi, avec des intensités lumineuses plus grandes que $200 \mu \mathrm{mol}$ photon. $\mathrm{m}^{-2} \cdot \mathrm{s}^{-1}$ et des photopériodes plus longues que $8 \mathrm{~h}$, en serre, nous avons obtenu des activités nitrate réductase de feuilles de blé de 3 à $6 \mathrm{j}$ aussi élevées que les plus fortes mesurées ici à 8-10 j.

En conclusion, les tests de faculté germinative peuvent être faits avec de l'eau jusqu'à 3 j; jusqu'à ce stade, la semence semble autonome vis-à-vis de l'alimentation minérale. Avec 55 $\mu \mathrm{mol}$ photon. $\mathrm{m}^{-2} \cdot \mathrm{s}^{-1}$, les tests de faculté germinative sont améliorés en «milieu clos» par la présence de $\mathrm{KNO}_{3} 20$ mmol.t-1 entre 3 et $6 \mathrm{j}$; si la durée de croissance est supérieure, l'activité nitrate réductase des plantules de blé est stoppée au stade de $6 \mathrm{j}$ en "milieu clos" et ne répond pas à l'augmentation d'intensité lumineuse.

Cependant les concentrations en protéines solubles enregistrées en "milieu clos" n'augmentent de manière importante que sous une intensité lumineuse plus grande que celle employée au SNES. Pour que la croissance et l'activité nitrate réductase continuent à augmenter après $10 \mathrm{j}$, une alimentation plus équilibrée et en apport journalier, sous une intensité lumineuse supérieure au point de compensation sont souhaitables.

On peut également remarquer que la concentration en $\mathrm{KNO}_{3}$ utilisée dans ce travail (20 mmol..$^{-1}$ ) et celle employée (60 mmol. $\mathrm{I}^{-1}$ ) par Disa et al $(1982,1985)$ sont très élevées. Dans les champs, les semences peuvent rencontrer 1 mmol. I $^{-1}$ d'après les estimations de la concentration dans une bonne terre végétale (Heller, 1984). De plus, les résultats récents obtenus par Devienne et al (1991) montrent que des concentrations inférieures à $1 \mathrm{mmol} . .^{-1}$ suffisent à obtenir une croissance et une teneur en azote maximales des jeunes plantules de blé.

\section{RÉFÉRENCES}

Abrol YP, Sawhney SK, Naik MS (1983) Light and dark assimilation of nitrate in plants. Plant Cell Environ 6, 595-599

Andrews M (1986) The partitioning of nitrate assimilation between root and shoot of higher plants. Plant Cell Environ 9, 511-519

Andrews M, De Faria SM, Mclnroy SG, Sprent JI (1990) Constitutive nitrate reductase activity in the Leguminosae. Phytochemistry 29, 49-54 
Aslam M, Huffaker RC, Rains DW, Rao KP (1979) Influence of light and ambient carbon dioxide concentration on nitrate assimilation by intact barley seedlings. Plant Physiol 63, 1205-1209

Bazzigalupi O (1991) Effet du $\mathrm{KNO}_{3}$ appliqué au semis sur la germination et les premiers stades de développement chez trois espèces : soja (Glycine max L Mer), haricot (Phaseolus vulgaris $L$ ) et blé (Triticum aestivum L). Thèse Doctorat Institut National Agronomique Paris-Grignon

Bradford MM (1976) A rapid and sensitive method for the quantitation of microgram quantities of protein utilizing the principle of protein-dye binding. Anal Biochem 72, 248-254

Caboche M, Rouzé P (1990) Nitrate reductase: a target for molecular and cellular studies in higher plants. Trends Genet 6, 187-192

Chen TM, Ries SK (1969) Effect of light and temperature on nitrate uptake and nitrate reductase activity in rye and oat seedlings. Can J Bot 47, 341-343

Coïc $Y$, Lesaint C (1975) La nutrition minérale et en eau des plantes en horticulture avancée. Doc Techn SCPA 23, 1-22

Deroche ME (1983) Relations entre la photosynthèse et l'assimilation de l'azote. Bull Soc Bot Fr Actual Bot 1983, 85-98

Devienne F, Chaillou S, Mary B, Lamaze T (1991) Managing nitrogen supply allows maximum growth without excessive $N$ accumulation in wheat plants grown under controlled conditions. In: Plant science today ( $Y$ de Kouchkovsky, ed) INRA Editions, Versailles, 119, ISBN: 2-7380-0349-4

Disa S, Gupta A, Rajasekhar VK, Guha-Mukherjee S, Sopory SK (1982) Rhythmicity in nitrate reductase activity in wheat embryos during germination. New Phytol 92, 495-499

Disa S, Gupta A, Guha-Mukherjee S, Sopory SK (1985) Requirement for a long lag period for the induction of nitrate reductase in wheat (Triticum aestivum) embryos during germination. New Phytol 99, $71-80$

Duke SH, Duke SO (1984) Light control of extractable nitrate reductase activity in higher plants. Physiol Plant 62, 485-493

Gupta A, Disa S, Saxena IM, Sarin NB, GuhaMukherjee S, Sopory SK (1983) Role of nitrate in the induction of nitrite reductase activity during wheat seed germination. J Exp Bot 34, 396-404
Gupta AK, Sharma AK, Guha-Mukherjee S, Sopory SK (1988) Inhibition of nitrate reductase induction in germinating barley embryos by endosperm. Plant Sci 54, 141-145

Heller R (1984) Physiologie végétale. 1. Nutrition, Masson, Paris, ISBN 2-225-80326-9

Hewitt EJ, Cutting CV (1979) Nitrogen assimilation of plants. Acad Press, Londres ISBN 0-12-346360-2

ISTA (1985) International rules for seed testing. Annexes. Seed Sci Technol 13, 236

Lee RB (1980) Sources of reductant for nitrate assimilation in non-photosynthetic tissue: a review. Plant Cell Environ 3, 65-90

Long DM, Oaks A (1990) Stabilization of nitrate reductase in maize roots by chymostatin. Planta Physiol $93,846-850$

Minotti PL, Jackson WA (1970) Nitrate reduction in the roots and shoots of wheat seedlings. Planta 95, 36-44

Moureaux T, Leydecker MT, Meyer C (1989) Purification of nitrate reductase from Nicotiana plumbaginifolia by affinity chromatography using $5^{\prime}$ AMP. Sepharose and monoclonal antibodies. Eur J Biochem 179, 617-620

Pace GM, Volk RJ, Jackson WA (1990) Nitrate reduction in response to $\mathrm{CO}_{2}$-limited photosynthesis. $\mathrm{Re}$ lationship to carbohydrate supply and nitrate reductase activity in maize seedlings. Plant Physiol 92, 286-292

Pinto Contreras MP, Gaudillère JP (1987) Efficacité de la croissance du Blé lors du passage à l'autotrophie. Plant Physiol Biochem 25, 35-42

Rodgers CO, Barneix AJ (1988) Cultivar differences in the rate of nitrate uptake by intact wheat plants as related to growth rate. Physiol Plant 72, 121-126

Srivastava HS (1980) Regulation of nitrate reductase activity in higher plants. Phytochemistry 19, 725-733

Talouizte A, Guiraud G, Moyse A, Marol C, Champigny ML (1984) Effect of previous nitrate deprivation on ${ }^{15} \mathrm{~N}$-nitrate absorption and assimilation by wheat seedlings. J Plant Physiol 116, 113-122

Wallace W (1986) Distribution of nitrate assimilation between the root and shoot of legumes and a comparison with wheat. Physiol Plant 66, 630-636

Wray JL, Filner P (1970) Structural and functional relationships of enzyme activities induced by nitrate in barley. Biochem J 119, 715-725 